This is the peer reviewed version of the following article:

Preparing the genetic counseling workforce for the future in Australasia.

First published: 01 Feb 2021

which has been published in final form at

https://onlinelibrary.wiley.com/doi/epdf/10.1002/jgc4.1358

This article may be used for non-commercial purposes in accordance with Wiley Terms and Conditions for Self-Archiving." 


\section{Preparing the genetic counseling workforce for the future in Australasia}

Alison McEwen ${ }^{1}$ and Chris Jacobs ${ }^{1}$

Genetic Counseling, Graduate School of Health, University of Technology Sydney, New South Wales, Australia

Running head: The genetic counseling workforce of the future

Corresponding author: Associate Professor Alison McEwen

Email: Alison.McEwen@uts.edu.au

Telephone: +61 (2) 95147202 


\section{Abstract}

Current genetic counseling students will graduate into a workforce involving more opportunities, diversity and uncertainty than any previous generation. Preparing the future genetic counseling workforce is a dynamic challenge, both for the profession and for educators.

The dominance of the medical model in the state funded Australian healthcare system creates a power imbalance between doctors and other health professionals. As a result, professional regulation to protect the public from harm in line with the USA, the UK and Canada, only became mandatory in 2019. Professional regulation has the additional benefit of enhancing professional standing and autonomy, enabling genetic counselors to help shape the future of genetic healthcare in Australia and New Zealand.

Within this rapidly evolving environment, we are establishing a new Masters' program and building a discipline of genetic counseling, working alongside other allied health professionals. Our program involves synchronous and asynchronous learning, greater accessibility, flexibility and, as we have learned in 2020 , reduction in disruption during a global pandemic. In this program, we foreground the inherent knowledge, skills and values of genetic counseling, shifting the focus from provision of genetic and genomic tests, to educating competent, person-centred, research enabled and culturally safe genetic counselors.

As educators, we have a responsibility to prepare students to embrace the uncertainties, challenges and potential of the genomic era, to seize the many possibilities that lie ahead, and to expand their thinking and vision. We invite our students to be courageous, to step into a deep exploration of their own identity, beliefs, understanding and experiences of oppression, power and privilege. We are pushing boundaries, challenging ourselves and our students to remain always open to possibilities. Equipping students with open eyes and listening ears may be the single most important thing we can do to prepare the genetic counseling workforce of the future to provide the best possible care.

Keywords: Genetic counseling, Genetic counselors, education, workforce 


\section{$\underline{\text { Introduction }}$}

We start by locating ourselves. Although we both now work on Gadigal land in Sydney, neither of us were born in Australia. We bring with us life and work experiences from Aotearoa New Zealand and the United Kingdom. We pay our respects to the Elders both past and present, acknowledging them as the traditional custodians of knowledge for the lands upon which we work.

Advances in genomics provide exciting opportunities for genetic counselors worldwide. To meet the challenging demands of these technologies, it is vital that the future genetic counseling workforce is highly skilled, knowledgeable, diverse and has the flexibility to adapt to future changes. Preparing students to embrace the opportunities afforded by these advances challenges the genetic counseling profession to identify and focus on the unique skills and knowledge that sets genetic counselors apart from other healthcare professionals.

This paper describes the opportunities and challenges of developing a new discipline of genetic counseling and a Masters' program that aims to meet these goals within the relatively young and newly regulated genetic counseling profession across Australia and New Zealand. These two countries cover a vast area and have diverse populations yet share a professional membership organisation and a common training and certification pathway.

Here we detail our commitment to providing accessible, engaging and interactive genetic counselor education to increase diversity among those entering the profession, the creation of brave spaces to facilitate the development of effective person-centred genetic counseling practice and the importance of facilitating education that encourages listening and resilience in future generations of genetic counselors. Our experience of providing future-focused genetic counselor education across two discrete health systems may inform the development of training programs elsewhere.

\section{Genetic counseling in Australia and New Zealand}

Australia is an affluent country with densely populated main centres close to coastal areas and huge expanses of bush and desert areas in between. Aboriginal and 
Torres Strait Islanders are the oldest living culture in the world and have lived, worked and cared for the land in Australia for millennia. More recently, successive waves of immigration have made some Australian cities among the most diverse in the world with huge social inequality (ACOSS 2018). Healthcare in Australia is funded by a national public health insurance scheme called Medicare and administered by several layers of government. Importantly, genetic counselors are not yet covered by Medicare which means that much genetic counseling practice is overseen by a medical practitioner.

New Zealand was settled by Māori and more recently by British and other immigrants, particularly from the Pacific. Aotearoa, New Zealand has a founding document, the Treaty of Waitangi, which enshrines principles of partnership, participation and protection in law. Healthcare in New Zealand is provided by a government funded public health system that includes funding for the provision of genetic counseling. There is currently no genetic counselor education program located in New Zealand and until now, students have moved to Australia to study.

The first genetic counseling training program was established in Newcastle, Australia in 1995 shortly followed by several other programs. These programs were embedded in medical schools and their location and mode of delivery required students to relocate, limiting equity and diversity within the profession. In Australasia (a term used to include both Australia and New Zealand), genetic counseling training and certification is administered by the Human Genetics Society of Australasia. Competencies required for certification include communication, counseling, critical thinking and reflective practice (HGSA, 2019a). Participation in regular career-long counseling supervision and reflective practice are requirements for ongoing certification in Australasia.

The Australian Government has recently invested heavily in the implementation of genomics into healthcare and this is now being rolled out state by state (Australian Government, 2020). The New Zealand Government has also invested in a number of nationally important genomic initiatives, placing Te Ao Māori (Māori worldviews) at the centre of funded projects (Genomics Aotearoa, 2020). The arrival of genomics is a disruptive change, bringing both opportunity and uncertainty as roles for genetic health professionals shift and adjust to technological advancements. 
Alongside the changes brought about by the genomic era, genetic counselors in Australia and New Zealand are facing further change as professional regulation is implemented. Regulation contributes to the safety of the public and enhances the professional standing of genetic counselors. Professional regulation has been a long term goal for genetic counselors in Australasia. In 2018 an application to the National Alliance of Self Regulating Health Professionals was accepted and regulation was implemented in October 2019 (HGSA, 2019b).

Significant changes in professional standing, while important, have the potential to disrupt the status quo, creating uncertainty, fatigue and the perception of threat among genetic counselors and other health professionals. The Human Genetics Society of Australasia is undertaking further work to ensure the varying roles genetic counselors have and the ways in which they fulfil the requirements for regulation when working in non-clinical roles are recognised.

\section{Genetic counselor education in a changing time}

Within this rapidly evolving environment, and in a country where populations are spread across vast distances, UTS Genetic Counseling began providing genetic counselor education.

The program at UTS is unique in Australasia, located in a Graduate School of Health, alongside other allied health disciplines, such as speech pathology, physiotherapy and clinical psychology, rather than in a medical school. Our positioning allows us to foreground the inherent knowledge, skills and values of genetic counseling and genetic counselors, and to educate person-centred, research enabled and culturally safe genetic counselors. Like the allied health disciplines we work alongside, we have a PhD program, ensuring we are contributing to the growing number of genetic counselors undertaking genetic counseling research.

At a time when new roles are emerging, genetic counselor education is a matter of importance for the profession, not just for those tasked with the responsibility of providing the education. Recognising this and drawing on participatory co-design principles (Bate and Robert, 2006) we included the voices of genetic counselors and other key stakeholders in the development of this program. Over $25 \%$ of the 
membership of the Australasian Society of Genetic Counselors participated in a series of teleconferences and discussions in 2017, sharing ideas to inform curriculum development. The need for profound changes in the diversity of people entering the profession and in the provision of culturally safe genetic counseling were among the key themes emerging from these discussions (McEwen, McLean \& Jacobs, 2019).

Genetic counseling education programs that allow students to remain located in their home state or country have the potential to increase the diversity of people entering the profession. Students remain within their own communities and support networks, continuing to meet pre-existing family and community responsibilities. Our program actively supports students to study from their home areas by utilising a combination of asynchronous interactive online learning and synchronous weekly, timetabled 'live and online' classes, supported with an on campus block each semester. This approach is facilitating access for students located across Australia, New Zealand and beyond. Students attend weekly face to face interactive workshops on Zoom throughout the program, where they apply their learning through participation in discussions, role plays, debates, presentations, and conversations with guests. The knowledge content is provided in an interactive online learning management system, facilitating engagement with learning materials, teachers and peers as students prepare for the weekly workshops. The restrictions arising as a result of the global pandemic in 2020 heighten the importance of making the best possible use of interactive technologies in genetic counselor education, enabling students learning to continue without disruption.

In a rapidly evolving landscape, it is important that educators prepare students to work in new and emerging roles. Utilising a design technique called 'making' that facilitates a collective imagining of possible and probable future states (Sanders \& Stappers, 2013), we have created opportunities for exploration of the evolving profession student genetic counselors are entering (McEwen, McLean \& Jacobs, 2019). 'Making' with an interdisciplinary Curriculum Advisory Committee, with students, and with practicing genetic counselors has generated thought-provoking and challenging conversations about the future of the profession, and what this means for genetic counseling education. 
Increasing diversity in genetic counseling requires a commitment to culturally safe practices within the education setting, ensuring students entering the profession are welcomed and recognised. In addition, a commitment to embedding cultural safety throughout the program to ensure graduates are well equipped to deliver culturally safe healthcare is crucial to foster ongoing change. Health providers and health systems play a significant role in creating and maintaining health inequities for Indigenous and minority groups (Anderson et al, 2016). Exposure to education that equips students to understand that health inequities are primarily due to unequal power relationships, an unfair distribution of the social determinants of health, unexamined privilege and institutional racism (Reid \& Robson, 2007), and explores their role in addressing these inequities, is an important role for education providers. Our program uses a language of cultural safety (Curtis et al, 2019) and challenges both staff and students to engage in an ongoing examination of our assumptions, biases and values, privilege and power, both during workshops and in supervision groups.

An understanding of the ways in which family, community, ethnicity, culture, education, healthcare and institutional and policy structures influence both clients of genetic counselors, and genetic counselors themselves, is fundamental to the practice of client-centred genetic counselors (Biesecker, Peters \& Resta, 2019). Ecological theory allows us to understand the ways in which the lives of people attending for genetic counseling are both supported and challenged by the layers of systems within which they live (Bronfenbrenner, 1996). Regarding individuals and families as "people-in-relationship' with our world, both inside and outside our skins' (Cook, 2012, p. 131), encourages us to take note of the broader structures, experiences and influences that are central to the provision of competent, clientcentred, genetic counseling care.

As educators, our understanding of students entering the profession is informed by ecological theory, providing a way to acknowledge and celebrate the cultures, worldviews, perspectives and experiences students bring into the program. Modelling an environment in which we are all both learners and experts will, we hope, create a similar approach to clinical practice in our graduates. Below, we provide examples of this approach from our curriculum. 


\section{Creating brave spaces in genetic counselor education}

Education, like genetic counseling is, fundamentally, an interaction between human beings (Biesta, 2015, Resta et al, 2006). As educators, we are privileged to have the opportunity to interact with students in ways that create passion and excitement for the field of study. In so doing, we acknowledge that deep learning occurs when students are enabled to take risks.

Creating safe spaces is a well-recognised ground rule for group work in education (Flensner \&Von der Lippe, 2019). Some educators are now questioning whether the goal of a safe space is idealistic, compatible, or appropriate (Arao \& Clemens, 2013; Cook-Sather, 2016). Rather than creating safe spaces, consider the possibilities for learning that occurs in a brave space - a space in which the educators have a 'responsibility to foster a learning environment that supports participants in the challenging work of authentic engagement' (Arao \& Clemens, 2013, p.138-139).

Framing education as an activity that occurs in a brave space supports the use of a narrative of courage with students - courage to accept the invitation to step outside their comfort zone, to come to know more about themselves and to know themselves in new ways, in order that they might come some way towards knowing each person they meet in their clinics.

We are delivering genetic counselor education in a dynamic young university committed to providing transformative, authentic, engaging educational experiences to a culturally and socially diverse population (UTS 2019). Developing and delivering a genetic counseling program in this environment allows us to create authentic, real world experiences with our students, as demonstrated by the following examples.

Example 1: In their first semester, students complete a subject that invites them to deepen their understanding of the lived experiences of the people with whom they will work. Drawing on our own learning from our colleagues in Indigenous health, we developed a subject using the Indigenous Australian framework of ways of knowing, being and doing (Martin, 2003). 'Ways of knowing' acknowledges the many ways in which people learn and gather knowledge, including observing, listening, reading, watching and waiting. 'Ways of being' acknowledges the ways we are in the world and the relational contexts in which we move through our world. 'Ways of doing' 
brings together ways of knowing and being in an expression of who we are in our world. Students are invited to go 'out and about' to learn about their local communities, and to engage with diversity through a range of media and experiences. Students come together for a virtual class each week in which they meet people with lived experience of inherited conditions, diversity, loss, and life, who share their experiences in conversation with the students. Collectively, these experiences are supported by participation in weekly small group reflective practice supervision facilitated by senior genetic counselors. Students create a record of learning through a series of reflective writing tasks.

Developing and delivering this subject, walking alongside students as they confront, explore and reflect upon their own identities and relationships with Indigenous Australia, with Mãori, with the diverse communities they live and work in, with lesbian, gay, bisexual, transgender, queer, intersex (LGBTQI+) communities, with those with disabilities and with people who have experienced the huge losses that can accompany inherited conditions is a huge privilege and an expression of what a brave space in education can lead to. Alongside the experiential learning, students participate in modules that introduce them to ecological theory and the theory and practice of cultural safety.

Student reflections indicate that they are aware that they still have a lot to learn, suggesting that a requirement to explore and reflect on the communities they are members of translates into an increased awareness of diversity of lived experience and a recognition of ongoing learning. Imagine if, as a global profession, we extended the requirement to engage with diversity beyond graduate education and into continuing professional development.

Example 2: The notion of brave spaces in education suggests a requirement for creation of a space in which students are simultaneously enabled to take risks, while being taken care of (Cook-Sather, 2016). Working with standardised clients in simulation activities encourages students to take risks and try new ways of communicating in a supportive learning environment.

The use of simulation as a learning activity (rather than an assessed task) draws on the experiential learning cycle (Kolb, 1984), inviting students to experience, review 
and reflect to deepen their learning and plan and participate in a second experience. Simulation provides students with authentic, 'real world' experiences, such as delivering difficult news that they may otherwise not experience during training. Working with senior genetic counselors in focus groups and interviews, a series of standardised clients, based on real clients, were developed for use with students. Standardised clients are played by actors, trained to respond as the client would. During full day workshops, starting in the first week of the program, students meet and work with standardised clients in clinical settings (Jacobs, Russell-Williams, \& McEwen, 2019). Academic staff and clinical educators work alongside the students and actors providing feedback so that students take the learning from one scenario into the next, applying what they have learnt. Students then take the learning from these experiences into the real world of clinical placement.

\section{$\underline{\text { Reflective practice supervision in genetic counseling education }}$}

As educators, we assist students to embrace the uncertainty, ambiguity and complexity of the genomic era, recognising the emerging opportunities. Participation in regular reflective-practice supervision fosters resilience in health professionals through development of coping skills, professional belonging and identity (Beddoe and Davys, 2016). Supervision provides a space for genetic counselors to make sense of the situations we encounter at work, to debrief, talk about our own sadness, anger or frustration, explore our evolving roles and celebrate our successes. Weekly small-group reflective-practice supervision facilitated by senior genetic counselors is embedded throughout our program, prioritising the development of resilient and autonomous practitioners.

\section{The role of listening in person centred genetic counseling practice}

Threaded throughout our program is a consistent and unwavering commitment to putting people at the centre of our work, to facilitate the development of a lifelong practice of truly client-centred genetic counseling in our graduates. We believe that attentive, thoughtful and reflective listening is central to providing effective personcentred genetic counseling (McEwen \& Jacobs, 2020). The practice of listening to understand is explicitly taught during the first year and woven throughout the program. 
Conceptualising genetic counseling as a place for listening challenges us to reconsider the way in which genetic counseling is sometimes framed. Do people come to genetic counseling seeking information, education, a test? Or do they in fact come seeking a place in which their story, their lived experience will be heard? And through the telling of that story, the hearing of it by an empathic, person-centred genetic counselor, will they take another step in their journey to make meaning of their experience?

Listening with intention, to empower individuals and families as they adapt to genetic information allows genetic counselors to support clients 'to use genetic information in a personally meaningful way' (Biesecker \& Peters, 2001, p. 194). It is our hope that as well as being experts in genomics, the graduates of our program, and the genetic counselors of the future, will be effective listeners, competent counselors, personcentred practitioners and the holders of the stories their clients will share as they seek to make meaning of the genetic condition present in themselves or their family, and the genomic information they may have.

If, as we educate the genetic counselors of the future, we equip them with open eyes to truly see the person in front of them and listening ears seeking to truly understand, we believe we will have prepared them well.

\section{Author Contributions}

Alison McEwen and Chris Jacobs conceptualized the work presented in this commentary. AM wrote the manuscript and both AM and CJ were involved in the editing and reviewing.

\section{Acknowledgements}

The work discussed in this paper has arisen out of the rich and dynamic, sometimes messy, always thought-provoking opportunity to develop and deliver a new Master of Genetic Counseling program. We are privileged to work with a team of senior genetic counselors and researchers and we thank them for the wealth of knowledge and experience they bring to our work. As educators, our work exists primarily for our students and we thank our two cohorts of students, who challenge us every day to think more deeply about our work and practice as genetic counselors. 
Conflict of interest: Alison McEwen and Chris Jacobs declare that they have no conflict of interest.

Human and Animal Studies: No human or animal studies were conducted by the authors for this article.

Data Availability Statement: This paper is a commentary, and as such there is no data held that supports the content of the paper.

ORCID

Alison McEwen https://orcid.org/0000-0001-8705-1190

Chris Jacobs https://orcid.org/0000-0002-9557-9080

\section{$\underline{\text { References }}$}

Anderson, I., Robson, B., Connolly, M., Al-Yaman, F., Bjertness, E., King, A., . . Yap, L. (2016). Indigenous and tribal peoples' health ( the Lancet -Lowitja institute global collaboration): A population study. The Lancet, 388(10040), 131-157. doi:http://dx.doi.org.ezproxy.lib.uts.edu.au/10.1016/S0140-6736(16)00345-7

Arao, B. and Clemens, K. (2013). From Safe Spaces to Brave Spaces: A New Why to Frame Dialogue Around Diversity and Social Justice. In Lisa Landreman (ed) The Art of Effective Facilitation, Sylus Publishing, Virginia.

Australian Council of Social Service (ACOSS), (2018). Inequality in Australia 2018. https://www.acoss.org.au/wp-content/uploads/2018/07/Inequality-in-Australia2018.pdf Retrieved 29 June 2020.

Australian Government, Department of Health, (2020). Genomics Health Futures Mission. https://www.health.gov.au/initiatives-and-programs/genomics-health-futures-mission Retrieved 17 June 2020. 
Bate, P. and Robert, G. (2006) 'Experience-based design: from redesigning the system around the patient to co-designing services with the patient.' Quality \& Safety in Health Care, 15, 307-310. Available at:

https://qualitysafety.bmj.com/content/15/5/307 (Accessed: 16 April 2018).

Beddoe, L. and Davys, A. (2016) Challenges in Professional Supervision: Current Themes and Models for Practice. London: Jessica Kingsley Publishers. ISBN-10: 1849054657

Biesecker, B. B., \& Peters, K. F. (2001). Process studies in genetic counseling: peering into the black box. Am J Med Genet, 106(3), 191-19 doi:10.1002/ajmg.10004

Biesecker B.B., Peters K.F. and Resta R. (2019). Advanced Genetic Counseling. Theory and Practice Oxford University Press, NY, USA

Biesta, G. J. (2015). Beautiful risk of education. London and New York: Routledge.

Bronfenbrenner, U. (1996). The ecology of human development experiments by nature and design. Cambridge, MA: Harvard University Press.

Cook, Ellen P. (2012). Understanding People in Context: The Ecological Perspective in Counseling, American Counseling Association, USA.

Cook-Sather, Alison. (2016). Creating Brave Spaces within and through Student-Faculty Pedagogical Partnerships, Teaching and Learning Together in Higher Education,18. http://repository.brynmawr.edu/tthe/vol1/iss $18 / 1$

Curtis, E., Jones, R., Tipene-Leach, D. et al. (2019). Why cultural safety rather than cultural competency is required to achieve health equity: a literature review and recommended definition. Int J Equity Health 18, 174. https://doi.org/10.1186/s12939-019-1082-3

Flensner, K.K. \& Von der Lippe, M. (2019). Being safe from what and safe for whom? A critical discussion of the conceptual metaphor of 'safe space', Intercultural Education, 30:3, 275-288, DOI: 10.1080/14675986.2019.1540102

Genomics Aotearoa, 2020 https://www.genomics-aotearoa.org.nz/ retrieved 3 March 2020 
Human Genetics Society of Australasia (HGSA), (2019a). Competency Standards for Genetic Counsellors https://www.hgsa.org.au/documents/item/10550 retrieved 7 June 2020

Human Genetics Society of Australasia (HGSA), (2019b) Genetic Counsellor Training, Certification and Regulation in Australia and New Zealand https://www.hgsa.org.au/resources/genetic-counselling retrieved 3 March 2020

Jacobs, C, Russell-Williams, S. \& McEwen, A. (2019). Making it real: students' experiences of simulation in the first week of genetic counselling education. Twin Research and Human Genetics, 22, p.393, doi:10.1017/thg.2019.81

Kolb, D.A. (1984). Experiential learning: experience as the source of learning and development. Englewood Cliffs, NJ: Prentice Hall.

Martin, K. \& Mirraboopa, B. (2003) Ways of knowing, being and doing: A theoretical framework and methods for indigenous and indigenist re-search, Journal of Australian Studies, 27:76, 203-214, DOI: 10.1080/14443050309387838

McEwen, A., Mclean, J., \& Jacobs, C. (2019). Reflections on the use of co-design to develop a genetic counselling curriculum fit for purpose for the genomic era: a case study. The Journal of Educational Innovation, Partnership and Change, 5(1). doi:http://dx.doi.org/10.21100/jeipc.v5i1.964

McEwen, A. and Jacobs, C. (2020). Who we are, what we do and how we add value: The role of the genetic counseling 'philosophy of practice' statement in a changing time. $J$ Genet Couns, 00: 1-7. DOI: 10.1002/jgc4.1308

Reid, P and Robson, B. (2007). Understanding Health Inequities. In Robson B, Harris R. Eds. Hauora: Māori Standards of Health IV. A study of the years 2000-2005. Te Rōpū Rangahau Hauora a Eru Pōmare: Wellington.

Resta, R., Biesecker, B. B., Bennett, R. L., Blum, S., Hahn, S. E., Strecker, M. N., \& Williams, J. L. (2006). A new definition of Genetic Counseling: National Society of Genetic Counselors' Task Force report. J Genet Couns, 15(2), 77-83. doi:10.1007/s10897-005-9014-3 
Sanders, E. and Stappers, P. (2013) The Convivial Toolbox: Generative Research for the Front End of Design. Amsterdam: BIS Publishers. ISBN-10: 9063692846

University of Technology Sydney, (2019), UTS 2027: Our Strategy. https://www.uts.edu.au/about/uts-2027-strategy/vision retrieved 10 March 2020 